\title{
Complete Genome Sequence Data of Nonpathogenic Strain Rhizobium vitis VAR03-1, a Biological Control Agent for Grapevine Crown Gall Disease
}

\author{
Yoshiteru Noutoshi, ${ }^{1, \dagger}$ Atsushi Toyoda, ${ }^{2}$ Tomoya Ishii, ${ }^{1}$ Kirara Saito, ${ }^{1}$ Megumi Watanabe, ${ }^{1}$ and \\ Akira Kawaguchi ${ }^{3}$ \\ ${ }^{1}$ Graduate School of Environmental and Life Science, Okayama University, Okayama, Japan \\ ${ }^{2}$ Department of Genomics and Evolutionary Biology, National Institute of Genetics, Mishima, Shizuoka, \\ Japan \\ ${ }^{3}$ Western Region Agricultural Research Center, National Agricultural and Food Research Organization \\ (NARO), Fukuyama, Hiroshima, Japan
}

\begin{abstract}
Crown gall disease in grapevine is caused by pathogenic strains of Rhizobium vitis with a tumor-inducing (Ti) plasmids. A nonpathogenic strain, VAR03-1 of $R$. vitis, has been isolated from the grapevine root of nursery stock and it was shown to act as a biological control agent to crown gall disease. Its disease-suppressive effect was observed even when it was coinoculated with the pathogen in a 1:1 ratio. Here, we present the complete genome data of $R$. vitis VAR03-1, assembled by sequencing reads obtained by both PacBio and Illumina technologies with annotation. This genome sequence could contribute to investigations of the molecular basis underlying the biocontrol activity as well as the root-colonization ability of this bacterial strain.
\end{abstract}

\section{Genome Announcement}

Rhizobium vitis (=Agrobacterium vitis, A. radiobacter biovar 3) VAR03-1 was isolated from surface-sterilized roots of nursery stock of grapevine (Vitis vinifera L. cv. Seto Giants) as a nonpathogenic strain of $R$. vitis during isolation of the causal agent of grapevine crown gall disease (Kawaguchi et al. 2005, 2007). In this paper, the nomenclature for Agrobacterium/ Rhizobium species follows the proposal by Young et al. (2001). This bacterial isolate was found to have a biological control activity that can suppress grapevine crown gall disease caused by tumorigenic (pathogenic) strains of $R$. vitis (Kawaguchi et al. 2005, 2007). Also, it was shown to suppress crown gall disease on tomato, rose, and apple caused by tumorigenic $R$. radiobacter (=tumorigenic $A$. tumefaciens, $A$. tumefaciens biovar 1 ) and tumorigenic $R$. rhizogenes (=tumorigenic $A$. rhizogenes, $A$. tumefaciens biovar 2), respectively, to a similar extent as nonpathogenic $R$. rhizogenes (=A. rhizogenes, $A$. radiobacter biovar 2 ) $\mathrm{K} 84$, a commercial biocontrol agent (Kawaguchi et al. 2008, 2012). R. rhizogenes K84 produces an antibiotic compound, named agrocin K84, that works as a structural analog of adenine nucleotide and prevents nucleic acid biosynthesis (Roberts et al. 1977). R. vitis VAR03-1

${ }^{\dagger}$ Corresponding author: Y. Noutoshi; noutoshi@okayama-u.ac.jp

Current address for Kirara Saito: Kyusyu Okinawa Agricultural Research Center, National Agriculture and Food Research Organization, Miyakonojo, Miyazaki, Japan

The author(s) declare no conflict of interest.

Accepted for publication 17 August 2020.

\section{Funding}

This work was financially supported by the Japan Society for the Promotion of Science through KAKENHI 16H06279 (Platform for Advanced Genome Science) and KAKENHI $17 \mathrm{H} 03778$ and KAKENHI $20 \mathrm{~K} 20572$ to A. Kawaguchi and Y. Noutoshi.

\section{Keywords}

antitumorigenic bacterial strain, biological control agent, crown gall disease, Rhizobium vitis 
showed antimicrobial activity to the tumorigenic strain of $R$. vitis, whereas $R$. rhizogenes K84 was nontoxic to this pathogen (Kawaguchi et al. 2005). This is consistent with the fact that $R$. rhizogenes $\mathrm{K} 84$ could not suppress crown gall disease in grapes. The antagonistic activity of $R$. vitis VAR03-1 was detected in the filtrate of the culture medium of $R$. vitis VAR03-1 (Saito et al. 2018). Because it was a large molecule ( $>100 \mathrm{kDa}$ ) with sensitivity to high temperature, the biocontrol activity of $R$. vitis VAR03-1 seems to be dependent on a protein or protein complex, unlike $R$. rhizogenes.

Rhizobiaceae is one of the families of order Rhizobiales and it consists of genera of Agrobacterium/Rhizobium, Ensifer/Sinorhizobium, and Candidatus Liberibacter. Most bacteria belonging to this family are rhizosphere-associated bacteria, which include nitrogenfixing endosymbionts, commensal endophytes, and pathogens. There are three biovars in the Agrobacterium/Rhizobium bacterial strains, based on physiological and biochemical properties that were used for classification apart from pathogenicity. To date, the whole-genome sequence of a pathogenic biovar 1 strain of $R$. radiobacter ( $=A$. tumefaciens) $\mathrm{C} 58$ has been reported (Goodner et al. 2001; Wood et al. 2001). After that, two additional genomes of the biocontrol strain $R$. rhizogenes $\mathrm{K} 84$ and another tumorigenic strain, $R$. vitis S4, which can infect grapevines, were determined with annotations (Slater et al. 2009).

Here, we performed whole-genome shotgun sequencing using PacBio and Illumina sequencing platforms on $R$. vitis VAR03-1. The genomic DNA was isolated using a QIAamp DNA Mini Kit (Qiagen, Hilden, Germany) and was sheared into fragments of sizes ranging from 20 to $50 \mathrm{kbp}$ with a g-tube device (Covaris Inc., Woburn, MA, U.S.A.). A SMRTbell library was prepared using a Template Prep Kit v1.0 (Pacific Bioscience, Menlo Park, CA, U.S.A.) according to manufacturer protocol. The sequencing library was size-selected using the BluePippin system (Sage Science, Beverly, MA, U.S.A.) with a minimum fragment length cutoff of $17 \mathrm{kbp}$. The final library was sequenced on the PacBio RSIl sequencer with SMRT cell v3 and P6-C4v2 chemistry, yielding a total of 45,261 reads (1.13 Gbp). The PacBio reads were assembled using the HGAP3 assembler program with default settings. In addition, genomic DNA was fragmented to an average size of $600 \mathrm{bp}$ with the DNA shearing system M220 (Covaris Inc.). A paired-end library was constructed with a TruSeq DNA PCR-Free Library Prep kit (Illumina, Inc., San Diego, CA, U.S.A.) and was size-selected on an agarose gel using a Zymoclean large fragment DNA recovery kit (Zymo Research, Irvine, CA, U.S.A.). The final library was run on the Illumina HiSeq 2500 sequencer with a read length of $250 \mathrm{bp}$. Illumina read sequences comprising $3,110,931 \times 2$ raw reads were used to improve the base quality of the assembled sequence obtained by the long-read sequences using the Pilon version 1.22 software tool. For annotation, DFAST, a DDBJ fast annotation and submission tool, was used (Tanizawa et al. 2016).

The final assembly of the genome is $5,571,018 \mathrm{bp}$ in length with zero gaps and a GC content of $57.6 \%$. There were, in total, 4,986 protein-coding sequences. The genome is divided into two chromosomes (circular) (sizes 3,604,775 and 1,156,266) and two plasmids (sizes 648,626 and 161,351 bp). Nine ribosomal RNAs and 51 transfer RNAs were located on chromosome 1, and three ribosomal RNAs and four transfer RNAs were on chromosome 2. In comparison with the genome size of the previously sequenced Agrobacterium/Rhizobium strains, which are 5,674,260 bp in tumorigenic $R$. radiobacter C58, 7,273,300 bp in $R$. rhizogenes $\mathrm{K} 84$, and 6,320,946 bp in $R$. vitis $\mathrm{S} 4$, the genome size of $R$. vitis VAR03-1 is the smallest. In the genome of $R$. vitis VAR03-1, no genes were found with an annotation of virulence-related functions.

This genome sequence will contribute to our understanding of the molecular basis of both the biocontrol activity and endophytic nature of $R$. vitis VAR03-1. In addition, it will be useful to investigate the genome evolution of Rhizobium/Agrobacterium.

This complete whole-genome sequence has been deposited in the DDBJ database under accession numbers AP023268 (chr1), AP023269 (chr2), AP023270 (pRvVAR031a), and AP023271 (pRvVAR031b) (PRJDB10104 for BioProject and SAMD00233295 for BioSample).

\section{Acknowledgment}

We are grateful for the financial support of the Japan Society for the Promotion of Science KAKENHI. We also thank the Platform for Advanced Genome Science and its scientists and 
supporting members for sequencing, management, financial supports, and excellent suggestions and comments.

\section{Literature Cited}

Goodner, B., Hinkle, G., Gattung, S., Miller, N., Blanchard, M., Qurollo, B., Goldman, B. S., Cao, Y., Askenazi, M., Halling, C., Mullin, L., Houmiel, K., Gordon, J., Vaudin, M., lartchouk, O., Epp, A., Liu, F., Wollam, C., Allinger, M., Doughty, D., Scott, C., Lappas, C., Markelz, B., Flanagan, C., Crowell, C., Gurson, J., Lomo, C., Sear, C., Strub, G., Cielo, C., and Slater, S. 2001. Genome sequence of the plant pathogen and biotechnology agent Agrobacterium tumefaciens C58. Science 294:2323-2328.

Kawaguchi, A., Inoue, K., and Ichinose, Y. 2008. Biological control of crown gall of grapevine, rose, and tomato by nonpathogenic Agrobacterium vitis strain VAR03-1. Phytopathology 98:1218-1225.

Kawaguchi, A., Inoue, K., and Nasu, H. 2005. Inhibition of crown gall formation by Agrobacterium radiobacter biovar 3 strains isolated from grapevine. J. Gen. Plant Pathol. 71:422-430.

Kawaguchi, A., Inoue, K., and Nasu, H. 2007. Biological control of grapevine crown gall by nonpathogenic Agrobacterium vitis strain VAR03-1. J. Gen. Plant Pathol. 73:133-138.

Kawaguchi, A., Kondo, K.-i., and Inoue, K. 2012. Biological control of apple crown gall by nonpathogenic Rhizobium vitis strain VAR03-1. J. Gen. Plant Pathol. 78: 287-293.

Roberts, W. P., Tate, M. E., and Kerr, A. 1977. Agrocin 84 is a 6-N-phosphoramidate of an adenine nucleotide analogue. Nature 265:379-381.

Saito, K., Watanabe, M., Matsui, H., Yamamoto, M., Ichinose, Y., Toyoda, K., Kawaguchi, A., and Noutoshi, Y. 2018. Characterization of the suppressive effects of the biological control strain VAR03-1 of Rhizobium vitis on the virulence of tumorigenic R. vitis. J. Gen. Plant Pathol. 84:58-64.
Slater, S. C., Goldman, B. S., Goodner, B., Setubal, J. C., Farrand, S. K., Nester, E. W., Burr, T. J., Banta, L., Dickerman, A. W., Paulsen, I., Otten, L., Suen, G., Welch, R., Almeida, N. F., Arnold, F., Burton, O. T., Du, Z., Ewing, A., Godsy, E., Heisel, S., Houmiel, K. L., Jhaveri, J., Lu, J., Miller, N. M., Norton, S., Chen, Q., Phoolcharoen, W., Ohlin, V., Ondrusek, D., Pride, N., Stricklin, S. L., Sun, J., Wheeler, C., Wilson, L., Zhu, H., and Wood, D. W. 2009. Genome sequences of three agrobacterium biovars help elucidate the evolution of multichromosome genomes in bacteria. J. Bacteriol. 191:2501-2511.

Tanizawa, Y., Fujisawa, T., Kaminuma, E., Nakamura, Y., and Arita, M. 2016. DFAST and DAGA: Web-based integrated genome annotation tools and resources. Biosci. Microbiota Food Health 35:173-184.

Wood, D. W., Setubal, J. C., Kaul, R., Monks, D. E., Kitajima, J. P., Okura, V. K., Zhou, Y., Chen, L., Wood, G. E., Almeida, N. F., Jr., Woo, L., Chen, Y., Paulsen, I. T., Eisen, J. A., Karp, P. D., Bovee, D., Sr., Chapman, P., Clendenning, J., Deatherage, G., Gillet, W., Grant, C., Kutyavin, T., Levy, R., Li, M. J., McClelland, E., Palmieri, A., Raymond, C., Rouse, G., Saenphimmachak, C., Wu, Z., Romero, P., Gordon, D., Zhang, S., Yoo, H., Tao, Y., Biddle, P., Jung, M., Krespan, W., Perry, M., Gordon-Kamm, B., Liao, L., Kim, S., Hendrick, C., Zhao, Z. Y., Dolan, M., Chumley, F., Tingey, S. V., Tomb, J. F., Gordon, M. P., Olson, M. V., and Nester, E. W. 2001. The genome of the natural genetic engineer Agrobacterium tumefaciens C58. Science 294:2317-2323.

Young, J. M., Kuykendall, L. D., Martínez-Romero, E., Kerr, A., and Sawada, H. 2001. A revision of Rhizobium Frank 1889, with an emended description of the genus, and the inclusion of all species of Agrobacterium Conn 1942 and Allorhizobium undicola de Lajudie et al. 1998 as new combinations: Rhizobium radiobacter, $R$. rhizogenes, R. rubi, R. undicola and R. vitis. Int. J. Syst. Evol. Microbiol. 51:89-103. 